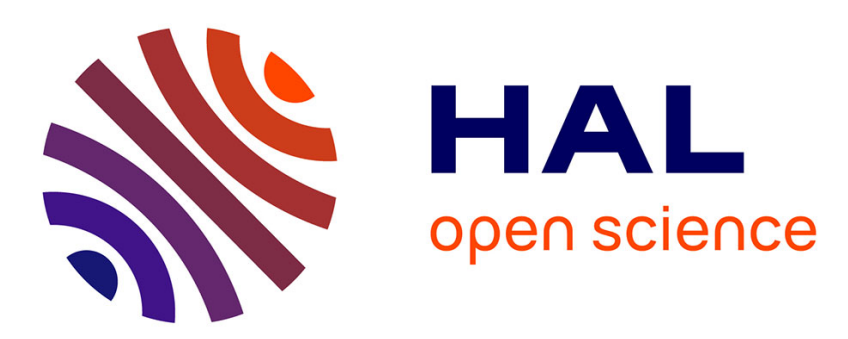

\title{
A low-cost high-precision measurement method of string motion
}

Jean-Loic Le Carrou, Delphine Chadefaux, Léonard Seydoux, Benoît Fabre

\section{To cite this version:}

Jean-Loic Le Carrou, Delphine Chadefaux, Léonard Seydoux, Benoît Fabre. A low-cost high-precision measurement method of string motion. Journal of Sound and Vibration, 2014, 333 (17), pp.3881-3888. 10.1016/j.jsv.2014.04.023 . hal-01461744

\section{HAL Id: hal-01461744 \\ https://hal.science/hal-01461744}

Submitted on 20 Nov 2017

HAL is a multi-disciplinary open access archive for the deposit and dissemination of scientific research documents, whether they are published or not. The documents may come from teaching and research institutions in France or abroad, or from public or private research centers.
L'archive ouverte pluridisciplinaire $\mathbf{H A L}$, est destinée au dépôt et à la diffusion de documents scientifiques de niveau recherche, publiés ou non, émanant des établissements d'enseignement et de recherche français ou étrangers, des laboratoires publics ou privés. 


\title{
A low-cost high-precision measurement method of string motion
}

\author{
J.-L. Le Carrou ${ }^{\mathrm{a}, \mathrm{b}, *}$, D. Chadefaux ${ }^{\mathrm{a}, \mathrm{b}}$, L. Seydoux ${ }^{\mathrm{a}, \mathrm{b}}$, B. Fabre ${ }^{\mathrm{a}, \mathrm{b}}$ \\ a Sorbonne Universités, UPMC Univ Paris 06, UMR 7190, LAM-Institut Jean le Rond d'Alembert, F-75005 Paris, France \\ ${ }^{\mathrm{b}}$ CNRS, UMR 7190, LAM-Institut Jean le Rond d'Alembert, F-75005 Paris, France
}

\begin{abstract}
The high-precision measurement of a string's motion requires either the use of an expensive apparatus or the development of a dedicated system. In this paper, a cheaper alternative based on opto-switch sensors combined with a suitable calibration is proposed. A sensitivity model requiring only two straightforward preliminary measurements to determine the parameters is presented. A comparison on a bench test between the opto-switch sensor and a high-speed camera has been performed. Results indicate that the calibrated opto-switch provides more accurate measurements of the string's motion in quasi-static as well as in dynamic states.
\end{abstract}

\section{Introduction}

To carry an experimentally based study of the string's vibrations, the choice or the design of a particular procedure depends on the string's properties as well as the global measurement context. Although researchers mostly use electromagnetic pickup [1], electrodynamic method [2] or electric field sensing methods [3-7] in the case of a steel string, these methods cannot be applied to other common string's materials such as gut or nylon. In this case, optical methods should be more appropriate to obtain the string displacement by an optical tracking device [8], by a photoelectric sensor [9] or using a high-speed camera coupled to image processing [10,11] or the string velocity through a laser doppler vibrometer [12]. Another optical method, less intrusive, expensive and cumbersome, consists in catching the shadow of the string by using an integrated opto-sensor [13], a photo-Darlington [14], or an analog position sensing detector [15], as well as an optoswitch system including an infrared emitting diode coupled to a photo-transistor [16]. The latter system has been used to measure the transverse $[17,18]$ and longitudinal $[19,20]$ string vibrations to analyse, for instance, nonlinear free vibrations [21] as well as chaotic vibrations [22] of a string. This system/sensor seems to be appropriate to measure the string displacement regardless of its material. However, it requires a difficult phase of calibration to determine the sensor sensitivity curve to properly convert the voltage signal to displacement and to take into account the inherent nonlinearity of the sensor [16].

A classical method to determine the sensitivity curve consists in moving the opto-switch system using a micrometer control while a string is positioned between the diode and the photo-transistor [23,24]. The output voltage is obtained with respect to the string position. This curve is a priori specific to each sensor and depends on the light environment. Moreover, the simultaneous analysis of the two transverse polarizations of a vibrating string at one or at multiple points along the

\footnotetext{
* Corresponding author.

E-mail address: jean-loic.le_carrou@upmc.fr (J.-L. Le Carrou).
} 
string needs multiple sensors and therefore multiple sensitivity curves. Considering for instance the investigation of a musical stringed instrument, the multiplication of the strings to measure will imply the highlighting of numerous sensitivity curves. This process is a tedious work that would benefit from a straightforward and accurate procedure.

In this paper, we propose a methodological framework valuable for the use of opto-switch sensors to measure precisely a string's motion. For this purpose, we first describe the sensor operation and a model of its sensitivity curve is proposed based on measurable parameters. Then, the use of a high-speed camera for measuring a string's motion is explained. Finally, a comparative analysis is performed between the investigated sensor and a high-speed camera in order to point out the advantages and the drawbacks of such a measurement method.

\section{Opto-switch sensor investigation}

Optoelectronic switches are mostly intended to develop non-contact switching by opaque objects passing through a slot between a light emitter (LED) and a light detector (photo-transistor). However, a careful geometrical adjustment of the slot allows the use of the simple functional transfer characteristic between the object position crossing the light beam and the output current of the light detector, providing that the object's motion is of the same order of magnitude as the diameter of the light beam. The sensor's functioning and its conditioning circuit are presented in Fig. 1.

\subsection{Opto-switch sensor's description}

In this subsection, the opto-switch sensor's description is illustrated with the OPB815L from OPTEK Technology Inc. It has been chosen because of its small size, its low-cost and its beam diameter which allows the study of most of the musical instruments' strings. However, the presented method can be generalized to other opto-switch sensors.

The on-chip optical arrangement creates an approximately parallel beam of about $1 \mathrm{~mm}$ diameter in the infrared range with a peak wavelength at $890 \mathrm{~nm}$. The light source is operated at a constant current of $8.2 \mathrm{~mA}$, fed through a $470 \Omega$ resistance from a stabilized $5 \mathrm{~V}$ voltage supply, as shown in Fig. 1 . The resulting power dissipated by the LED is less than $10 \mathrm{~mW}$, corresponding to 10 percent of the maximal power dissipation as described in the data-sheet. This low current value was chosen to offer maximum long term stability and to limit thermal reduction of LED efficiency.

The NPN photo-transistor is mounted on a traditional non-inverting common-collector (emitter follower) circuit, ensuring a low output impedance together with a high bandwidth. According to the data-sheet, a $3 \mathrm{~mA}$ emitter current is expected for full illumination of the photo-transistor for the LED polarization given above. Together with the same $5 \mathrm{~V}$ voltage supply on the collector as on the LED polarization branch, the $1 \mathrm{k} \Omega$ emitter resistance (see Fig. 1 ) allows a 3-0 V reading on the output while keeping the linear operating range of the transistor ( $2 \mathrm{~V}$ minimum collector-emitter voltage).

The emitter output is connected to an acquisition card through a $2 \mathrm{~m}$ coaxial cable yielding a $50 \mathrm{pF}$ capacitance. While the data-sheet does not give any specific indication, typical response time of photo-transistors such as the one used is about $\tau=10 \mu \mathrm{s}$. As a result, the bandwidth is limited by the rise time of the photo-transistor to a frequency of $f_{\text {cut }}=1 / 2 \pi \tau$, about $16 \mathrm{kHz}$.

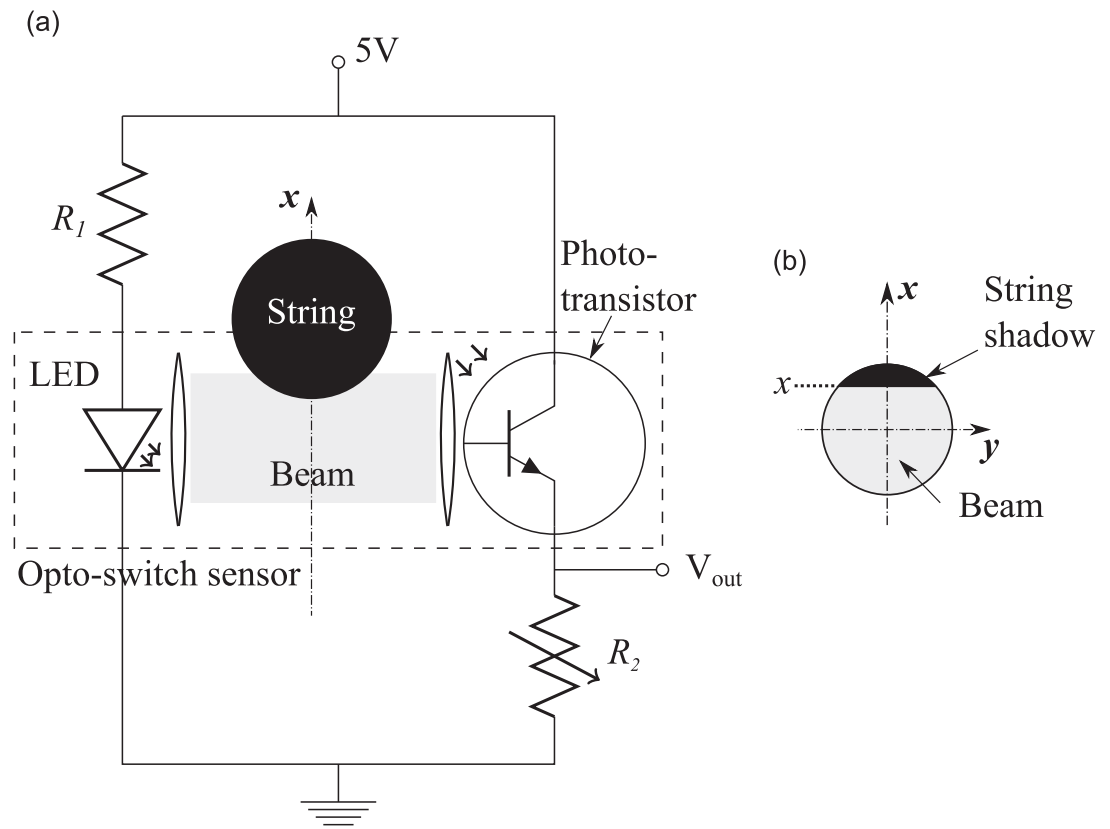

Fig. 1. (a) Electric circuit description with the opto-switch sensor. (b) Scheme of the sensor's beam on the photo-transistor while the string is oscillating. 


\subsection{Opto-switch sensor sensitivity's modeling}

In order to define the opto-switch sensor's calibration procedure, its sensitivity is modeled based on the photo-transistor and the string properties.

The luminous flux received by the photo-transistor can be decomposed as follows:

$$
\Phi_{p}=\Phi_{\mathrm{LED}}-\Phi_{S}+\Phi_{\mathrm{LE}} \quad \text { with } \Phi_{s}=\frac{A_{s}(x)}{A_{p}} \gamma \Phi_{\mathrm{LED}}
$$

where $\Phi_{\mathrm{LED}}, \Phi_{\mathrm{LE}}$ and $\Phi_{s}$ are emitted by the LED, by the light environment and absorbed by the string, respectively. $\gamma$ is the string's opacity, assumed to be homogeneous and $A_{s}(x)$ is the area of the string's shadow on the photo-transistor along the $x$ axis (see Fig. 1) of surface $A_{p}$.

Under the assumption of a homogeneous cylindrical infra-red beam and considering the photosensitive area circular and $\Phi_{p}$ proportional to the output voltage $V$ (see Fig. 1 ), we deduce

$$
V=V_{p}\left(1-\frac{A_{s}(x)}{A_{p}} \gamma\right)+V_{\mathrm{LE}}
$$

where $V_{p}$ is the output voltage with no string in a dark room and $V_{\mathrm{LE}}$ is the voltage produced by the light environment. The ratio $A_{s}(x) / A_{p}$ is calculated from the area of the circular segment produced by the string shadow at the $x$ abscissa as shown in Fig. 1. This leads to the theoretical expression of $V$ depending on the photo-transistor characteristics ( $r$ its radius and $\left.V_{p}\right)$, the light environment $\left(V_{\mathrm{LE}}\right)$ and the string's opacity $(\gamma)$ :

$$
\left.V=V_{p} \quad 1-\frac{\gamma}{2}+\frac{\gamma}{\pi} \arcsin \left(\frac{x}{r}\right)+\frac{\gamma x}{\pi r} \sqrt{1-\left(\frac{x}{r}\right)^{2}}\right)+V_{\mathrm{LE}}
$$

This equation can be re-written using $V_{\min }$ and $V_{\max }$ which are respectively the minimal output voltage (i.e. when the diode is covered by an opaque object) and the maximal output voltage (i.e. when the sensor's beam is not obstructed):

$$
\left.V=\left(V_{\max }-V_{\min }\right) 1-\frac{\gamma}{2}+\frac{\gamma}{\pi} \arcsin \left(\frac{x}{r}\right)+\frac{\gamma x}{\pi r} \sqrt{1-\left(\frac{x}{r}\right)^{2}}\right)+V_{\min } .
$$

Eq. (4) shows that the output voltage of the sensor is directly related to the string's displacement and to the sensor and string physical parameters. The photo-transistor radius $r$ is generally given in its data-sheet. However, for non-opaque strings, this radius is found to be 10-15 percent smaller because of the particular reflection properties of these strings. In order to estimate the parameters values $\left\{V_{\min }, V_{\max }, \gamma\right\}$, two measurements are carried out as follows: first, $V_{\min }$ and $V_{\max }$ are deduced from two straightforward measurements with an opaque object moving through the laser beam. Second, the string's opacity $\gamma$ is written as

$$
\gamma=\frac{V_{\max }-\min (V)}{V_{\max }-V_{\min }},
$$

where $\min (V)$ is the minimal output tension (for $x=-r$ ) when the string studied is moving in front of the photo-transistor. Note that as expected for an opaque string $\min (V)=V_{\min }$ and $\gamma=1$.

\subsection{Uncertainty}

The sensitivity of the sensor (see Eq. (4)) is estimated from physical parameters obtained by preliminary measurements. These parameters are thus tarnished by uncertainties which may affect the sensor accuracy. In order to quantify the latter, it is essential to compute the uncertainty on the sensors' sensitivity. From an analytical point of view, we estimate the uncertainty of the sensitivity curve with the uncertainty propagation method [25]. Assuming that each variable has independent random errors, it leads to

$$
u_{c}^{2}(V)=\left(\frac{\partial V}{\partial r}\right)^{2} u(r)^{2}+\left(\frac{\partial V}{\partial \gamma}\right)^{2} u(\gamma)^{2}+\left(\frac{\partial V}{\partial V_{\min }}\right)^{2} u\left(V_{\min }\right)^{2}+\left(\frac{\partial V}{\partial V_{\max }}\right)^{2} u\left(V_{\max }\right)^{2}
$$

where $u(r), u(\gamma), u\left(V_{\max }\right)$ and $u\left(V_{\min }\right)$ are respectively the uncertainties of $r, \gamma, V_{\max }$ and $V_{\min }$. Each partial derivative can be developed from Eq. (6) as follows:

$$
\begin{gathered}
u_{c}^{2}(V)=\left(V_{\max }-V_{\min }\right)^{2} \frac{4 x^{2}}{\pi^{2} r^{4}}\left(1-\frac{x^{2}}{r^{2}}\right) \gamma^{2} u^{2}(r)+\left(\frac{\gamma}{\pi} \arcsin \left(\frac{x}{r}\right)+\frac{x \gamma}{\pi r} \sqrt{1-\frac{x^{2}}{r^{2}}}+1-\frac{\gamma}{2}\right)^{2} u^{2}\left(V_{\max }\right)+\cdots \\
\left(\frac{1}{\pi} \arcsin \left(\frac{x}{r}\right)+\frac{x}{\pi r} \sqrt{1-\frac{x^{2}}{r^{2}}}-\frac{1}{2}\right)^{2}\left[\left(V_{\max }-V_{\min }\right)^{2} u^{2}(\gamma)+\gamma^{2} u^{2}\left(V_{\min }\right)\right]
\end{gathered}
$$


(a)

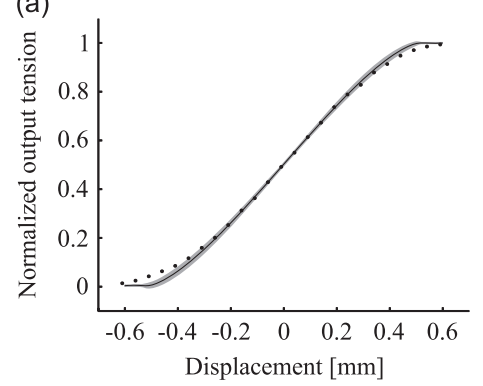

(b)

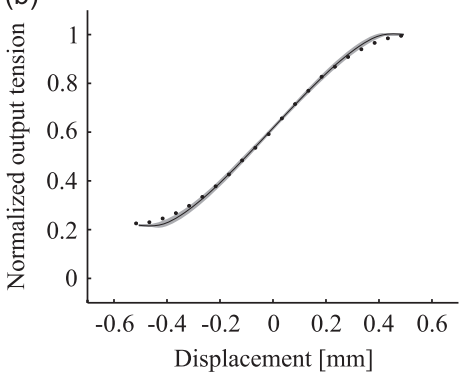

(c)

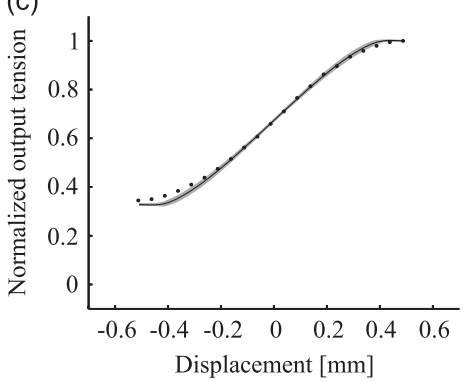

Fig. 2. Experimental (dotted line) and theoretical (black line, from Eq. (3)) sensor's sensitivity for three string materials: (a) steel, (b) nylon and (c) gut. Expanded uncertainty (grayed area) with 95\% confidence interval obtained by using Eq. (6).

where $u(\gamma)$ is obtained from Eq. (5) as

$$
\left.\left.\left.u(\gamma)^{2}=\frac{1}{V_{\max }-V_{\min }}-\frac{V_{\max }-\min (V)}{\left(V_{\max }-V_{\min }\right)^{2}}\right)^{2} u\left(V_{\max }\right)^{2}+\frac{1}{\left(V_{\max }-V_{\min }\right)^{2}}\right)^{2} u(\min (V))^{2}+\frac{V_{\max }-\min (V)}{\left(V_{\max }-V_{\min }\right)^{2}}\right)^{2} u\left(V_{\min }\right)^{2},
$$

while $u\left(V_{\max }\right), u(\min (V))$ and $u\left(V_{\min }\right)$ are estimated by repeatability measurements. Finally, two methods are available to determine $u(r)$ : either through a minimization process between the experimental data and the theoretical model in Eq. (4), or according to the error estimation of the photo-transistor radius from the sensor response curve provided in the datasheet.

\subsection{Experimental results}

The sensor's sensitivity and uncertainties can now be computed. Fig. 2 shows sensitivity curves obtained for three kinds of string material: steel, nylon and gut. Additionally, measurements performed using a micro-metrics translation system are pictured. Experimental data are found to be very close to theoretical results for each kind of string, mainly around $-0.35 \mathrm{~mm}$ to $0.35 \mathrm{~mm}$ where the expanded uncertainty is minimal. Beyond these values, the model is not accurate enough since it does not take into account the edge effects of the light on the string. Besides, the computed uncertainties are entirely consistent with the experimental data in the validity range. Note that the transparency of the string reduces the sensitivity of the sensor.

For accurate measurements, the optimum position of the sensor along the string length can easily be determined. Indeed, the theoretical sensor's sensitivity gives an insight into the usable range of magnitude. This indication combined with the maximal amplitude of the string's transverse vibration along its length (estimated through a classical string's vibration theory [28]) determines the ideal sensor position, often close to the boundary conditions of the string.

\section{Camera investigation}

\subsection{Object's motion detection}

The high-speed camera gives access to the string motion in the two-dimensional plane (defined by the framing directions) or in the three-dimensional space by using a mirror as in a previous study [10]. The position of a given point on the string is detected automatically through image processing. The area of interest is defined in the first image by the user, creating the initialization template. Its contour is detected through active-contour modeling [26], allowing the estimation of its center's position. A new template, corresponding to the initial one, is then searched in the next image through a blockmatching algorithm model [27]. This process is recursively repeated for all images.

Movements along the $x$ - and $y$-axis are directly obtained through the camera framing. They are written as

$$
\begin{aligned}
& x_{c}=\tilde{x} K \cos \theta_{1}, \\
& y_{c}=\tilde{y} K \cos \theta_{2},
\end{aligned}
$$

where $\tilde{x}$ and $\tilde{y}$ are the string's position in pixels with respect to $\vec{e}_{x}, \vec{e}_{y}$, respectively. $\theta_{1}$ and $\theta_{2}$ are the relevant angular rotations as shown in Fig. 3. $\mathrm{K}$ is the conversion factor from pixel to meter.

\subsection{Uncertainties}

The standard uncertainties associated to the measurands $x_{c}, y_{c}$ are referred to as $u_{c}\left(x_{c}\right), u_{c}\left(y_{c}\right)$. Assuming that each variable has independent random errors, we can apply the propagation of uncertainties formula [25] to Eqs. (9) and (10). We 
(a)

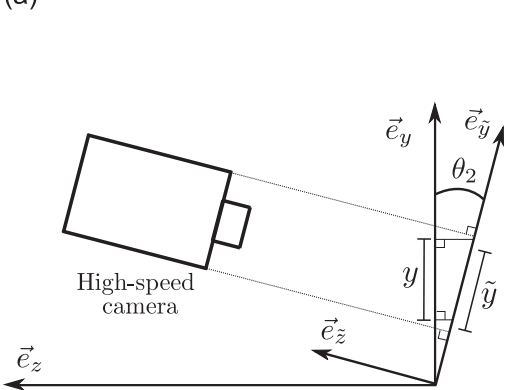

(b)

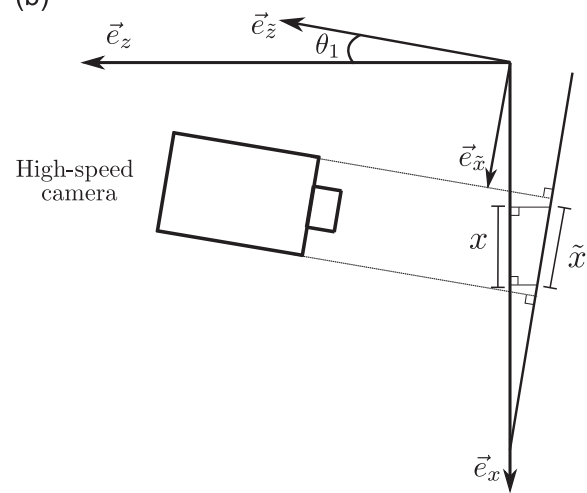

Fig. 3. High-speed camera setup referred to the $(y, z)$ plane (a) and on the $(x, z)$ plane (b).

can estimate the set of parameters $\left\{\theta_{1}, \theta_{2}, K\right\}$ and their combined standards measurement uncertainties through geometrical estimation and repeatability uncertainties obtaining $u_{c}\left(\theta_{1}\right)=u_{c}\left(\theta_{2}\right)=0.35^{\circ}$ and $u_{c}(K)=8 \times 10^{-6} \mathrm{~m} / \mathrm{px}$.

To estimate the uncertainty induced by the image processing $\left(u_{c}(\tilde{x})\right.$ and $\left.u_{c}(\tilde{y})\right)$, several sources of error have to be considered: the accuracy of the algorithm implemented, its sensitivity to the signal-to-noise ratio in images, and the influence of the user defining the area of interest in the first image. The robustness of the detection method for the peak signal-to-noise ratio (PSNR) was evaluated within the addition of a white noise with zero mean and finite variance (from 0 to 0.27 ) to the image's sequence. In the present case, since raw sequences are preprocessed in order to improve the contrast in images, the mean PSNR value during the tracking object process is of about $20 \mathrm{~dB}$, which corresponds to $u_{c}(\tilde{x})=u_{c}(\tilde{y})=0.05 \mathrm{px}$. Finally, the measurement uncertainties may be written as

$$
\begin{aligned}
& u_{c}\left(x_{c}\right)^{2}=\left(\cos \theta_{1} K u_{c}(\tilde{x})\right)^{2}+\left(\cos \theta_{1} \tilde{x} u_{c}(K)\right)^{2}+\left(\sin \theta_{1} K u_{c}\left(\theta_{1}\right)\right)^{2}, \\
& u_{c}\left(y_{c}\right)^{2}=\left(\cos \theta_{2} K u_{c}(\tilde{y})\right)^{2}+\left(\cos \theta_{2} \tilde{y} u_{c}(K)\right)^{2}+\left(\sin \theta_{2} K u_{c}\left(\theta_{2}\right)\right)^{2} .
\end{aligned}
$$

Experimental parameters $\theta_{1}, \theta_{2}$ and $K$ can thus be minimized to improve measurements uncertainties. As the two angles $\theta_{1}$ and $\theta_{2}$ are dependent on the experimental setup and the conversion factor $K$ is related to the image resolution, the uncertainties are directly dependent on the experimental setup.

\section{Comparison of the two measurement methods}

In this section, a comparison between an opto-switch sensor properly calibrated by using the method proposed in Section 2 and a high-speed camera is performed. After a description of the experimental setup, the results are discussed.

\subsection{Experimental procedure}

To compare the proposed methods for measurement of the transverse motion of strings, an experimental procedure has been devised based on the measurement of a harp wound metal string (of $1.56 \mathrm{~mm}$ diameter) fixed on a bench test through an opto-switch sensor (OPB815L-OPTEK Technology Inc.) and a high-speed camera (Phantom V9-Vision Research) set at $15037 \mathrm{fps}$ for a resolution of $256 \mathrm{px} \times 256 \mathrm{px}$ while the string oscillates at around $90 \mathrm{~Hz}$.

The experimental setup is shown in Fig. 4. In order to properly measure the transverse vibration magnitude $x$ in the valid range as explained in Section 2.4, the opto-switch sensor is positioned at $y_{s}$, close to the string's boundary. The string of length $L$ is plucked at $y_{0}=L / 2$. To obtain the highest resolution, the high-speed camera focuses on a point with a higher displacement magnitude, referred to as $y_{c}$.

For comparison purposes, the data from both measurements need to be interpreted in terms of a string vibration model, since measurements are carried out at two different positions along the string. Therefore, the string motion is divided into two time phases: the plucking and the free oscillation. It has been shown [10] that in the case of a finger plucking a harp string, the string shape can be assumed to be a simple triangle during the plucking phase time, when the finger is pulling the string from its rest position. For this time phase, the string motion measured at $y_{s}$ can be easily transferred at $y_{c}$ homothetically.

The free oscillation is the dynamical part of the string's motion. Assuming that we have a flexible string of uniform and linear density $\rho_{l}$, stretched to a tension T fixed at its ends, the transverse vibrations in the $x$-direction at $y_{s}$ is [28]

$$
x\left(y_{s}, t\right)=\sum_{n=1}^{\infty}\left(a_{n} \cos \left(2 \pi f_{n} t\right)+b_{n} \sin \left(2 \pi f_{n} t\right)\right) \Phi_{n}\left(y_{s}\right) e^{-\alpha_{n} t}
$$


where $t$ is the time, $f_{n}=n c / 2 L$ are the eigen-frequencies, $\alpha_{n}$ are the damping coefficients, $\Phi_{n}$ are the modal shapes, and $a_{n}$ and $b_{n}$ are the modal amplitudes. Eq. (13) can be rewritten under the form of the exponential sinusoidal model:

$$
x\left(y_{s}, t\right)=\sum_{n=1}^{k} A_{n} \sin \left(2 \pi f_{n} t+\Psi_{n}\right) e^{-\alpha_{n} t},
$$

where all parameters $\left(A_{n}, f_{n}\right.$ and $\left.\alpha_{n}\right)$ can be estimated using the ESPRIT algorithm (Estimation of Signal Parameters via Rotational Invariance [29]). Eventually, the string's displacement at $y_{c}$ can be computed as follows:

$$
x\left(y_{c}, t\right)=\sum_{n=1}^{\infty}\left(\frac{A_{n} \cos \Psi_{n}}{\Phi_{n}\left(y_{s}\right)} \cos \left(2 \pi f_{n} t\right)-\frac{A_{n} \sin \Psi_{n}}{\Phi_{n}\left(y_{s}\right)} \sin \left(2 \pi f_{n} t\right)\right) \Phi_{n}\left(y_{c}\right) e^{-\alpha_{n} t},
$$

with $\Phi_{n}(y)=\sin (\pi n y / L)$ for a string fixed at both ends, and compared to the direct measurement obtained by the highspeed camera.

\subsection{Results}

Fig. 5 presents the transverse string's motion at the same point $y_{c}$ measured both by the high-speed camera and by the properly calibrated opto-switch sensor. The voltage delivered by the opto-switch sensor at $y_{s}$ is in the range [0.22 V-0.77 V],

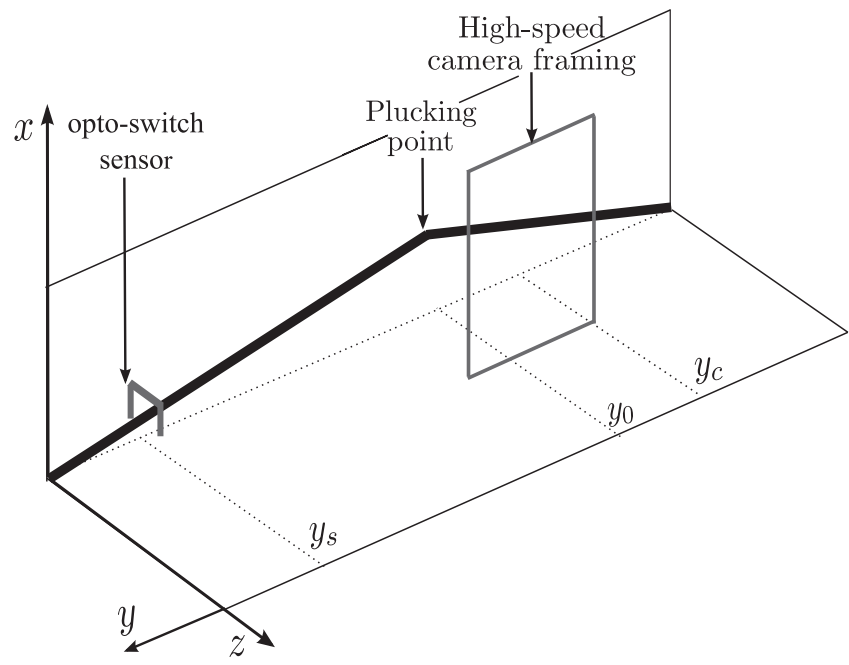

Fig. 4. Experimental setup to compare string's motion measurement through high-speed camera and opto-switch sensor.

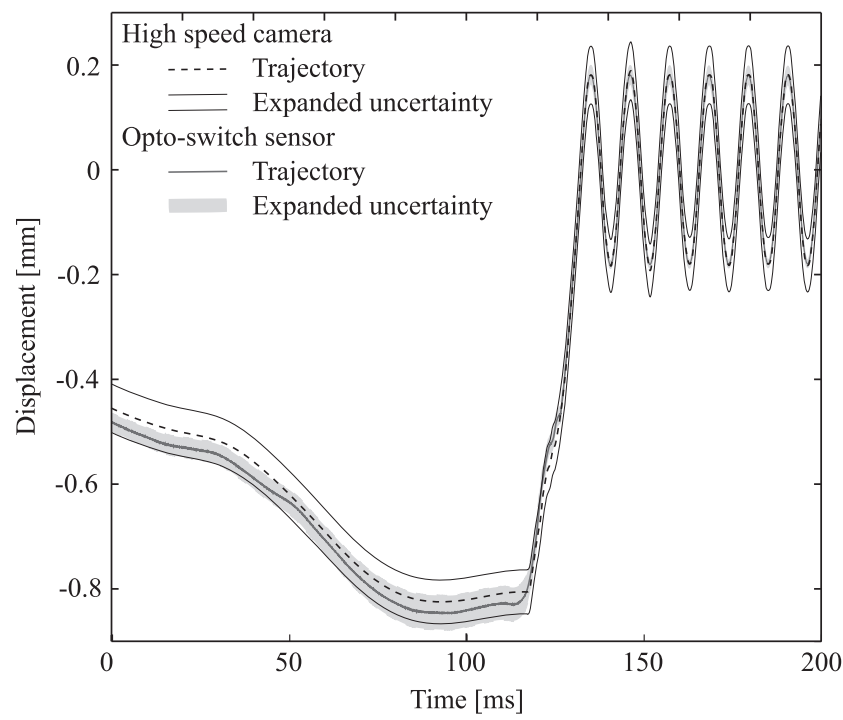

Fig. 5. String's transverse displacement estimated with the high-speed camera and the opto-switch sensor. The reported uncertainty represents a 95\% confidence interval. 
corresponding to a magnitude vibration of $450 \mu \mathrm{m}$, which is particularly adapted for the theoretical sensor's sensitivity. The trajectory shown in Fig. 5 from the opto-switch sensor is obtained thanks to the procedure explained in Section 4.1.

Fig. 5 only presents the last $120 \mathrm{~ms}$ of the plucking action and the 6 first oscillation periods. The black and gray dotted lines describe the trajectories measured respectively with the high-speed camera and the opto-switch sensor. The trajectories are provided with their associated uncertainties computed as explained in Sections 2.3 and 3.2 with a 95 percent confidence interval. As expected, in both cases, uncertainties increase with the deviation of the string from its rest position. In Fig. 5, the reported uncertainty of the trajectory measured with the opto-switch sensor is found to be within the uncertainty of the trajectory measured with the high-speed camera $\left(u_{c}(x)^{\max }=4\right.$ percent). This result indicates that the opto-switch sensor is more accurate than the high-speed camera to measure string motion in a static as well as in a dynamic regime.

Let us now compare the advantages and the drawbacks of the two measurement procedures relative to the application. First, as shown in Fig. 5, the accuracy of the opto-switch sensor, with the sensitivity model proposed in this paper, is better than that of a high-speed camera in both static and dynamic phases. Indeed, as no additional equipment is needed to measure the transverse motion of a string, there are less sources of error. Although the opto-switch sensor is a point measurement system, the full spatial responses may be inferred in combination with a string model. Besides, the optoswitch sensor is clearly less invasive than the high-speed camera setup because no additional light is needed. This is of great interest to perform non-invasive measurements in a realistic functioning context. Nevertheless, the opto-switch sensor has to be positioned for optimal performance taking into account the maximum string vibration magnitude as previously explained.

\section{Conclusion}

This paper describes a straightforward procedure to precisely measure the transverse motion of a musical instrument's string in both static and dynamic regimes. The experimental method consists in using an opto-switch sensor with a proper calibration phase. The sensitivity curve is determined based on a physical model of the sensor in which parameters are estimated through two straightforward measurements. Performances of this procedure are compared to those of an experimental procedure based on a high-speed camera. Results indicate that the opto-switch sensor is more accurate both in the static phase and in the dynamic phase than the high-speed camera. Finally, an application of this sensor to a harp string transverse motion and the determination of the initial conditions of its free oscillation is provided. This precise and non-invasive sensor is a valuable tool to perform relevant string vibration analyses, especially in the case of musical instruments.

\section{Acknowledgments}

The authors acknowledge Hélène Moingeon, Christian Ollivon for their technical support during this project and Olivier Jacquet for his help during the experimental setup.

\section{References}

[1] Q. Leclere, J.-L. Le Carrou, P.-A. Roiron, Caractérisation expérimentale de la réponse d'une corde vibrante sollicité par un plectre, Proceedings of French Congress of Mechanics, Besancon, 2011.

[2] A. Askenfelt, E.V. Jansson, From touch to string vibrations. III: string motion and spectra, Journal of the Acoustical Society of America 93 (4) (1993) 2181-2196.

[3] G. Weinreich, Coupled piano strings, Journal of the Acoustical Society of America 62 (6) (1977) 1474-1484.

[4] N. Giordano, J.P. Winans II, Plucked strings and the harpsichord, Journal of Sound and Vibration 224 (3) (1999) 455-473.

[5] J. Pakarinen, M. Karjalainen, An apparatus for measuring string vibration using electric filed sensing, Proceedings of Stockholm Music Acoustics Conference, Stockholm, Sweden, 2003.

[6] X. Boutillon, Model for piano hammers: experimental determination and digital simulation, Journal of the Acoustical Society of America 83 (2) (1988) $746-754$.

[7] F.S. Gillian, S.J. Elliott, Measurement of the torsional modes of vibration of strings on instruments of the violin family, Journal of Sound and Vibration 130 (2) (1989) 347-351.

[8] O. O'Reilly, P.J. Holmes, Non-linear and non-periodic vibrations of a string, Journal of Sound and Vibration 153 (3) (1992) $413-435$.

[9] A. Alsahlani, F.B. Mathis, R. Mukherjee, Vibration suppression of a string through cyclic application and removal of constraints, Journal of Sound and Vibration 331 (2012) 4395-4405.

[10] D. Chadefaux, J.L. Le Carrou, B. Fabre, L. Daudet, Experimentally based description of harp plucking, Journal of the Acoustical Society of America 131 (1) (2012) 844-855.

[11] N. Plath, High-speed camera displacement measurement (HCDM) Technique of string vibrations, Proceedings of Stockholm Music Acoustics Conference, Stockholm, Sweden, 2013.

[12] J.-L. Le Carrou, F. Gautier, N. Dauchez, J. Gilbert, Modelling of sympathetic string vibrations, Acta Acustica United with Acustica 91 (2) (2005) $277-288$.

[13] M. Pavlidou, A Physical Model of the String-Finger Interaction on the Classical Guitar, PhD thesis, University of Wales, Cardiff, UK, 1997.

[14] C.G.B. Baker, C.M. Thair, C.E. Gough, A photo-detector for measuring resonances of violin strings, Acustica 44 (1980) 70.

[15] Y. Achkire, A. Preumont, Optical measurement of cable and string vibration, Shock and Vibration 5 (1998) 171-179.

[16] R. Hanson, Optoelectronic detection of string vibration, The Physics Teacher 25 (1987) 165-166.

[17] R.J. Hanson, H.K. Macomber, A.C. Morrison, M.A. Boucher, Primarily nonlinear effects observed in a driven asymmetrical vibrating wire, Journal of the Acoustical Society of America 117 (1) (2005) 400-412. 
[18] A. Chaigne, J. Chabassier, N. Burban, Acoustics of pianos: physical modeling, simulations and experiments, Proceedings of Stockholm Music Acoustics Conference, Stockholm, Sweden, 2013.

[19] A.R. Lee, M.P. Rafferty, Longitudinal vibrations in violin strings, Journal of the Acoustical Society of America 73 (4) (1983) $1361-1365$.

[20] M.P. Rafferty, A.R. Lee, Fast decay of longitudinal vibrations in violin strings, Proceedings of Stockholm Music Acoustics Conference, Stockholm, Sweden, 1983.

[21] C. Gough, The nonlinear free vibration of a damped elastic string, Journal of the Acoustical Society of America 75 (6) (1984) 1770-1776.

[22] T.C.A. Molteno, N.B. Tufillaro, Torus doubling and chaotic string vibrations: experimental results, Journal of Sound and Vibration 137 (2) (1990) $327-330$.

[23] R.J. Hanson, J.M. Anderson, H.K. Macomber, Measurements of nonlinear effects in a driven vibrating wire, Journal of the Acoustical Society of America 96 (3) (1994) 1549-1556.

[24] H. Wright, The Acoustics and Psychoacoustics of the Guitar, PhD Thesis, University of Wales, Cardiff, UK, 1996.

[25] John R. Taylor, An Introduction to Error Analysis-The Study of Uncertainties in Physical Measurements, University Science Books, Sausalito, California, 1982.

[26] T.F. Chan, L.A. Vese, Active contours without edges, IEEE Transactions on Image Processing 10 (2) (2001) 266-277.

[27] S.A. El-Azim, An efficient object tracking technique using block-matching algorithm, Proceedings of National Radio Science Conference, Alexandria, Egypt, 2002, pp. 427-433.

[28] P.M. Morse, Vibration and Sound, McGraw-Hill Book Company, 1948468 pp.

[29] R. Roy, A. Paulraj, T. Lailath, ESPRIT-a subspace rotation approach to estimation of parameters of cisoids in noise, IEEE Transactions on Acoustics, Speech and Signal Processing 34 (5) (1986) 1340-1342. 